\title{
The Illinois River Project and Oklahoma's Quest for Environmental Quality
}

\author{
Mark Meo \\ Science and Public Policy, University of Oklahoma, Norman, OK
}

$\mathrm{T}$ The Illinois River is a popular tourist and recreation attraction that flows from northwestern Arkansas through northeastern Oklahoma, and holds the distinction of being the first river designated wild and scenic by the State of Oklahoma. Each year more than 180,000 persons float the Illinois River by canoe, raft, or kayak. An estimated 350,000 enjoy swimming, fishing, camping, hiking, birding, and hunting opportunities. The river provides drinking water for Tahlequah and Watts, irrigates farms and nurseries, and is the habitat for several state and federal threatened and endangered species (Bality et al. 1998). Though the economy is based primarily on tourism, a substantial amount derives from agriculture, especially from poultry farming and cattle ranching, and from plant nurseries, forestry, gravel and limestone mining. The city of Tahlequah, which hosts Northeastern State University and the Cherokee Nation tribal government, also helps anchor the regional economy (Bality et al. 1998).

Since the late 1980s Arkansas and Oklahoma have been joined in various disputes over the environmental quality of the boundary-traversing river and its wider watershed. Controversy first arose from the discharge of municipal wastewater into the Illinois River by the city of Fayetteville, Arkansas. Since the river is state-designated as wild and scenic in Oklahoma, increased wastewater discharges by Arkansas created significant controversy, which, in turn triggered a lawsuit by Oklahoma. This legal action culminated in a U.S. Supreme Court decision in 1992 that resolved the conflict in Arkansas' favor. Heightened interest in the welfare of the watershed has continued since then. A comprehensive river basin management plan was approved by the Oklahoma Scenic River
Commission in 1998, but concerns over municipal discharges and uncontrolled nutrient loading, particularly phosphorus, from poultry litter (waste) into the Illinois River continued to grow.

In 1997, the U.S. Environmental Protection Agency, in cooperation with the National Science Foundation, awarded a Water and Watersheds research grant to a team of researchers at the University of Oklahoma, Oklahoma State University, and the University of Oklahoma Health Sciences Center. Since the research orientation was defined by the granting agencies to be on stakeholderbased decision making, a team was recruited and organized that could analyze the views and values of various watershed stakeholders within a context of management alternatives for the basin's land and water resources. The research award funded an initial three-year study (subsequently extended) of development impacts in the Illinois River watershed to test a protocol designed to foster watershed management policy that was effective, efficient, implementable, feasible, and acceptable. The project's goal was to provide agency decision makers and managers with a stakeholder-based perspective of what issues were believed to be important, and how those issues could be addressed through a negotiated management protocol. In the sections below, I discuss the organization of the Illinois River project, what its research methods and findings were, and how they were used by decision makers and educators after the project ended. A more detailed discussion can be found in Meo et al. (2002) and the literature cited below.

\section{The Illinois River Project}

Since the project put an emphasis on procedural legitimacy that would address the range of values 
held by stakeholders in the region, the cooperation and close coordination of the Oklahoma Scenic Rivers Commission administrator, Ed Fite, was key to success. As principal investigator, I recruited a multi-disciplinary research team that had the expertise and ability to address all of the identified impacts in a thorough and collaborative manner. The team included an environmental policy analyst (M. Meo), a hydrologist (B. Vieux), and an architect (B. Pettus) from the University of Oklahoma (OU); a political scientist with expertise in small-group decision making (W. Focht), an environmental economist ( $\mathrm{K}$. Willett), and a recreation specialist (L. Caneday) from Oklahoma State University; and a limnologist (R. Lynch) from the University of Oklahoma Health Sciences Center. The architect was an expert in computerbased visualization, a relatively new technique that the team planned to use to integrate disparate technical aspects of the project and present them to stakeholders in an understandable and engaging way during negotiation sessions. In addition, two environmental philosophers (E. Sankowski and Z. Trachtenberg) from OU were invited to join the research team to observe, participate, and assess the progress of the novel interactive negotiation protocol.

With Oklahoma Scenic Rivers Commission (OSRC) administrator Fite's assistance, we identified 15 stakeholder categories that were geographic location- and policy-specific for subsequent contact and analysis. The protocol exercise was divided into three phases. In Phase I, the research team performed a baseline assessment of existing environmental impacts in the basin. This phase provided policy makers, who were categorized as a separate stakeholder class, a basis upon which they could determine distinct policy alternatives for river basin impact management. In the second phase, the research team first performed alternative-specific impact assessments that were then presented to stakeholders in three focus group sessions for their reactions. Afterwards, the assessments and stakeholder reactions were presented at a second policy maker workshop to help them reach consensus on a preferred impact management strategy. In Phase III, the preferred strategy was again assessed for expected reductions in impacts and presented at a public meeting. This assessment and stakeholder reactions were then presented to policy makers for their negotiation of a final revision of the impact management policy. At the conclusion of the study, a basinwide telephone survey of opinion concerning the acceptability of the final policy preference served as an evaluation mechanism to test the efficacy of the protocol.

The Phase I baseline impact assessment was divided into four assessment types.

1. Physical Impact Assessment. Impacts from basin activities that could cause erosion, sedimentation, streambank instability, streambed scouring, debris obstruction, channel braiding, river course changes, and other physical impacts in the river corridor.

2. Biological Impact Assessment. Impacts of basin activities that could affect wildlife habitat, species abundance and diversity, water quality, and human health.

3. Economic Impact Assessment. The status of the regional economy and the factors that affected it. This included an evaluation of all economic sectors and the development of an economic model, with multipliers, that could be used to predict changes.

4. Sociopolitical Impact Assessment. Policy makers' and stakeholders'judgments of existing impacts to social, cultural, aesthetic, and community resources. Stakeholder preferences for impact management were also identified.

The baseline assessments were integrated and converted into a computer-based decision support tool: an interactive, multimedia, impact visualization platform - the Watershed Management Decision Support System (or WMDSS). Aerial and ground-based photography was obtained and, when combined with Geographic Information Systems (GIS) base maps were used as background for the visualizations. These backgrounds were overlaid with the results of the baseline assessments and animated to produce visual images that simulated impacts. In addition, an influence diagram that depicted and explained the relationships among elements of impact causal pathways was included. The WMDSS was designed and built to allow policy makers and stakeholders to interactively query the system to gain a better understanding of the range of impacts that affected the river and surrounding watershed, and to engage in meaningful discussions about alternative future 
actions and activities. In this respect, the WMDSS was designed to function as a vehicle for research integration and information organization that could be used as a broader public education tool, rather than as a real-time decision support tool.

\section{Project Methods and Findings}

\section{Physical Impact Assessment}

Physical impacts were assessed through a modeling approach. Researchers developed and applied a distributed parameter hydrological model that related ecological and water quality impacts to stakeholder management practices, and was used to assess potential land use alternatives. Modeling results were rendered into visual imagery that facilitated communication with stakeholders concerning the implications of development and management practices in the basin. A second task relied on distributed parameter hydrological modeling to relate land management alternative impacts to water quality. The modeling process required spatially variable data including digital elevation, land cover, soil properties, land use, and many other distributed data sets on the Illinois River Basin. Over twenty-five different maps were assembled using Arc-view GIS software. Among water quality parameters, nutrients (i.e. nitrates and phosphorus) were the main concern in the basin. Nitrogen and phosphorus concentrations at three USGS river gauging sites were used to analyze the relationship between phosphorus concentration and discharge for 1990-1998. The established relationship was used to estimate the daily phosphorus concentration at upstream and downstream cities in the watershed. Further analysis showed that 76 to 88 percent of the longterm loading to the river came from runoff during high-flow seasons.

Another objective was the development of a Distributed Runoff nonpoint Pollution model (DRIP) that integrated GIS and nonpoint pollution modeling techniques using deterministic water quantity and quality components. The model was used to predict the total mass of phosphorus that would be delivered by surface runoff to the outlet of the catchment during a storm event. The model was applied to the Illinois River Basin upstream of Tahlequah for a storm event that occurred June $9-12,1995$. The model predicted the total mass of phosphorus at the outlet of the watershed to within 8 percent error for this event. Sensitivity of the mass loading of phosphorus to land use changes was tested. A 50 percent reduction in the influent phosphorus concentration from runoff resulted in a 50 percent reduction in terms of riverine concentrations and loadings. This 1:1 relationship is expected for a conservative contaminant entering the waterway with no attenuation or absorption in the channel or to sediment. The corresponding reduction in riverine phosphorus concentration and loading due to reduced fertilizer and manure was investigated and incorporated into the economic assessment. For additional information, see Vieux and Moreda (2002a, 2002b, 2002c, 2003).

\section{Biological Impact Assessment}

Our team developed visual renderings of turbidity and aquatic species assemblages to help communicate the risks to biological communities in the Illinois River watershed and Lake Tenkiller. In this situation, different stakeholder groups held different views on the meaning and importance of the same information. A significant challenge was the development of visual risk communication materials that were scientifically valid yet meaningful to all stakeholder groups. Some stakeholder groups (regulatory and scientific) viewed biological communities more as indicators of water quality conditions while others (fishermen and floaters) viewed them, especially the fish community, more as a harvestable resource. Others viewed considerations such as biodiversity as ethical issues. Historical diatom and benthic macroinvertebrate data were treated by similar methods. Diatom photographs for all species were scanned into the database and several diversity indices were calculated. High quality photographs/ drawings of the benthic macroinvertebrate community were harder to obtain, especially at the species level where differences are imperceptible to most viewers; therefore, visual representations were obtained at the genus level. Six diversity indices were calculated for these data. The biological quality of these small streams ranged from very poor to good; therefore, an adequate representation of the range of biotic condition was available for presentation.

One of the primary effects of nutrient loading in 
the Illinois River Basin has been the eutrophication of Lake Tenkiller. Areas of the lake that were once used by scuba divers are now too turbid for this activity because of algal-induced turbidity. In addition, hypolimnetic oxygen deficits have been occurring in the upper end of the lake. Based upon an earlier EPA Clean Lakes study, it was calculated that a 70-80 percent reduction in phosphorous loading would be necessary to decrease algal growth. Data were entered into the database in graphical format (total phosphorous vs. secchi disk, chlorophyll vs. phosphorous, chlorophyll vs. nitrogen) to be interfaced with nutrient loading information from watershed nutrient and hydrologic modeling efforts.

In addition to the three USGS gauging stations, the Oklahoma Scenic Rivers Commission conducts sampling at seven locations along the river and monitors for turbidity, total nitrogen, nitrate, ortho-phosphate, and total phosphorous. These data were gathered and graphed, with indications of appropriate water quality standards for the period of record, although the time frame is too short to make conclusions about water quality trends. Unfortunately, no discharge data are available with these measurements; however, these data strengthen the database by providing information at sites more familiar to river visitors at public access sites. Discharge monitoring reports for the past five years were obtained for the 4 OPDES permitted point source dischargers in the basin. These data (DO, BOD, $\mathrm{pH}, \mathrm{TSS}$, $\mathrm{NH}_{3}$, TP, discharge, fecal coliform) were graphed together with permit values to give an indication of the influence of point source discharges on water quality.

A significant concern of many stakeholders was the effects of animal waste on water quality, specifically the over 45 million poultry produced annually. In order to address these concerns, a measure of the relative magnitude of poultry and cattle waste in the basin was developed. Data on human and animal numbers were gathered from the U.S. Census Bureau and the Natural Resource Conservation Service. Additional data were gathered from NRCS and other sources concerning the amount of nitrogen and phosphorous produced by humans and animals. Based upon phosphorous and nitrogen production, we calculated that the poultry and cattle in the basin were equivalent to approximately 5 and 15 million people, respectively. An additional concern has been the effects of septic tanks on water quality. We calculated that 72 percent of human generated waste is discharged to septic tanks, which elevated the concern over their influence; however, the amount of nutrients produced by humans was at most 1-2 percent of that produced by animals (see Table 1).

\section{Economic Impact Assessment}

Economic impact analysis in each of the relevant Illinois River Basin regions was conducted in three stages: regional baseline impact assessments, regional option-specific assessments, and refined basin-wide assessments. It was assumed that tourism/recreational activity is an important contributor to the economic base of the Illinois River Basin (Bality et al. 1998). The tourism/ recreational linkages in local economy were estimated with the input-output model IMPLAN (Impact Analysis for Planning). With respect to recreational activities, Illinois River floaters were surveyed regarding their knowledge of the environment, management strategies employed, and satisfaction with their respective recreation experiences. Visitors were contacted on outfitters' buses prior to their float experience and were asked to complete an "ecological and river management knowledge" test. Data were coded and analyzed. Findings were subsequently utilized by the sociopolitical assessment team and the economic assessment team.

The impact of tourism/recreational activity is driven by expenditures which constitute a component of final demand for the region. The changes in these final demand expenditures were

Table 1. Sources of Nutrients in the Illinois River Basin (1997).

\begin{tabular}{lrrrrrr}
\hline & Broilers & Layers/Pullets & Cattle & Dairy & Humans & Nurseries \\
\hline Number & $43,977,664$ & $1,948,775$ & 89,114 & 10,909 & 93,641 & 3 \\
Nitrogen (kg/yr) & $17,645,725$ & $1,092,538$ & $5,100,764$ & $1,091,682$ & 478,780 & 12,738 \\
Phosphorous (kg/yr) & $5,129,954$ & 404,898 & $1,765,470$ & 196,074 & 47,878 & 1,174 \\
\hline
\end{tabular}


the key for motivating the economic impacts which were of concern in this research. The first task in quantifying these linkages was to estimate the magnitude of recreational expenditures. This was done in the following manner. First mean expenditures per person per trip were multiplied by annual visits to the relevant sites of the Illinois River Basin. The estimated total trip expenditures associated with a site are allocated to economic sectors in the local economy of IMPLAN by using a "bridge matrix." The economic indicators analyzed included total gross output, employment, employee compensation, property income, value added and indirect business taxes.

Nurseries and greenhouse operations also constituted a significant portion of the region's economic activity. Likewise, this industry may have played an important role in establishing the environmental conditions within the Illinois River Basin area. The industry linkages in the local economy were estimated by using the inputoutput model IMPLAN. The economic indicators analyzed include total gross output, employment, employee compensation, property income, value added, and indirect property taxes. This analysis indicated that tourism generated roughly $\$ 7.7$ million in total output for Cherokee County, as well as 249 jobs and \$2.4 million in income. Direct, indirect, induced, and total economic impacts were estimated for the three Oklahoma counties for visitor declines of 5,000,10,000, and 25,000. Of the three counties, the majority of impacts were felt in Cherokee County.

Broiler production has also become an important component of the economy in the Illinois River Basin region. A by-product of this industry is poultry litter. This litter is an important source of crop nutrients, but high concentrations of litter applications raise concerns about the environmental impacts of increased nitrate, phosphorus, and bacteria levels in water supplies. The loss of phosphorus in runoff from agriculture is thought to be an important source of eutrophication (see Table 2).

A review of the literature on poultry waste management revealed a number of important shortcomings. A common feature of existing model structures was that poultry litter was treated as a factor of production. Thus the model structures were concerned with the derived demand for poultry litter and the nutrients embedded in the litter. In contrast, the supply sources of poultry litter were treated in an exogenous fashion. An overriding concern was that the opportunity cost of adjustments to environmental policies might have been overstated. The shortcomings noted above were addressed by developing a modeling framework that used an integrated approach to incorporate broiler feeding and production decisions with decisions on disposing of poultry waste. A key element of waste disposal is cropping activity. The presence of cropping decisions serves the role of establishing a derived demand for poultry litter within the region. The cropping decisions also include specifications for commercial fertilizer use. The initial database for this model was developed and a feasible solution was obtained.

The optimization model was used to examine a set of policy scenarios based on defining a set of limits placed on the maximum level of phosphorus allowed in the Illinois River Basin. Once each of the above situations was established, the model was implemented to reflect a number

Table 2. Broiler Production, Litter Generation, and Related Nutrients for 1997.

\begin{tabular}{lrrrrr}
\hline County & $\begin{array}{r}\text { Number of } \\
\text { Broilers }\end{array}$ & $\begin{array}{c}\text { Live Weight } \\
\text { (tons) }\end{array}$ & $\begin{array}{c}\text { Tons of } \\
\text { Litter }\end{array}$ & $\begin{array}{c}\text { Tons of } \\
\text { Nitrogen }\end{array}$ & $\begin{array}{c}\text { Tons of } \\
\text { Phosphorus }\end{array}$ \\
\hline Adair (OK) & $12,147,732$ & 28,486 & 16,618 & 499 & 415 \\
Cherokee (OK) & $3,336,028$ & 7,822 & 4,564 & 137 & 114 \\
Delaware (OK) & $28,493,904$ & 66,818 & 38,981 & 1,169 & 974 \\
Benton (AR) & $113,132,954$ & 265,296 & 153,872 & 4,616 & 3,847 \\
Washington & $102,347,152$ & 240,004 & 139,202 & 4,176 & 3,480 \\
(AR) & & & & & \\
Total & $259,457,770$ & 608,426 & 353,237 & 10,597 & 8,830 \\
\hline
\end{tabular}


of situations called "futures." The futures were based on assumed changes for broiler production. These different futures were applied to the three counties in Oklahoma while assuming that the number of broiler production units in Arkansas counties remained constant. The various futures were defined as follows: High growth $=75 \%$; Medium growth $=50 \%$; Low growth $=25 \%$; Status quo (same as the base case); Low decline = $-25 \%$; Medium decline $=-50 \%$; and High decline $=-75 \%$. The limits set on phosphorus ranged from a high of 153 tons to a series of lower tonnages that corresponded to concentrations of $.05 \mathrm{mg} / \mathrm{l}$ ( 56 tons), $.037 \mathrm{mg} / 1$ (30 tons), and .02mg/l (22.4 tons). The $.037 \mathrm{mg} / 1$ constraint was the focus of earlier negotiations between Oklahoma and Arkansas. For additional information, see Willett et al. (2006).

\section{Sociopolitical Impact Assessment}

The socio-political assessment addressed two major components of the research project. The first involved the identification of stakeholders' concerns about the Illinois River, preferences for river basin management, and knowledge of the causes and effects of river basin activities. The second responsibility was to design and conduct an asynchronous policy dialogue between policymakers and stakeholders that yielded consensus policy outputs that are judged legitimate by both the participants and the larger stakeholder population.

In the first phase of the project, the sociopolitical assessment team completed the baseline assessment. Interviews of stakeholders, interested parties, and policy makers were completed in Spring, 1999. Three rounds of interviews were conducted involving 270 participants. In the first round, 150 face-to-face interviews were held with participants residing, recreating, doing business, or having regulatory jurisdiction over activities in the Illinois River Basin. In this first round, an extended discussion of participant concerns about activities adversely affecting the Illinois River Basin were conducted and tape-recorded. This discussion was followed by another discussion about the participants' preferences for the management of these impacts, which was also tape-recorded. Each participant was then asked to complete a short demographic questionnaire that explored age, gender, race, education, occupation, length and type of residence in the basin and in one of the eight geographic study regions in the basin, sources of information about impacts, trustworthiness of these sources, political activity in the basin, activities engaged in, and economic stake in the basin.

Next, each participant was asked to complete Likert scale items inquiring into trust and preferences for policy making. These scales covered trust of local, state, and federal government officials to make policy in the basin, trust of experts and each other, perceived degree of certainty surrounding relevant facts about river basin impacts, and perceived degree of controversy that existed among stakeholders about preferred river basin impact management policies. Each participant was then asked to rank order eight alternative policy making strategies, ranging from technocratic expert-based policy making to fully participatory third-party facilitated policy making. Finally, the participants were asked to conduct a cognitive mapping exercise in which they recorded onto cards their concerns about river basin impacts, indicate the relative confidence they had in their knowledge about each impact concern, and then arranging the cards in such as manner as to reflect their conceptualization of river basin impacts.

In the second round, 120 additional face-to-face interviews were conducted using Q methodology (Focht 2002). Two Q sorts were obtained from each interview and both were tape-recorded. In the first, the participants sorted a 58-item Q sample of statements abstracted from round one interview discussions about impact concerns. In the second, the participants sorted a 47-item Q sample of statements abstracted from round-one interview discussions about impact management preferences. Each participant was asked to explain each Q sort to ensure that the interviewer understood the bases for item placements.

In the third round, 60 more face-to-face interviews were conducted using mental modeling. Based on an expert model (influence diagram) of physical, biological, economic, social, and legalpolitical impacts developed in the first year of the project, the participants were interviewed to ascertain their knowledge of the impacts, their causes, their effects, and their inter-relationships. Responses to questions about these impacts were coded in real time. A short card ranking, in which three pre-defined impact concerns were rank- 
ordered by each participant on separate cards, was also accomplished (Focht 2002). Finally, minutes of Oklahoma Scenic River Commission meetings over the last 16 years were content analyzed.

The questionnaire data, Likert scale data, and card ranking data were analyzed using descriptive statistics. The responses obtained from the Likert scales (trust, uncertainty, and controversy) were combined with the card ranking data (policy making strategies) to empirically test a prescriptive model of policy making legitimacy developed earlier by W. Focht. The tape-recorded open-ended discussions on impact concerns and impact management preferences were professionally transcribed. The research team conducted a content analysis of these transcripts to identify and quantify frequencies of concerns and management preferences. Data on concerns were also compared with the data analysis of concerns identified in the cognitive mapping exercises. A good correspondence between the two analyses was found, lending support to their validity.

The cognitive maps were entered into databases and then qualitatively coded as categories of concerns. Descriptive statistics were computed for impact concerns among participants (and later compared to content analysis findings, as stated above). Group membership of individual concerns included within clusters of concerns within maps were also analyzed using hierarchical aggregative cluster analysis (Focht 2002). Seven distinct clusters of concerns were identified. From these clusters, an aggregate cognitive map was defined that identified how participants conceptualized impacts in the basin. Later, a congregate map of impacts that demonstrated how concerns were related to each other in a social system was prepared. Q sort data was correlated and factor analyzed using PCQanal software (Focht 2002). Five factors emerged from the analysis of concern sorts and four factors emerged from the analysis of management preference sorts. Each factor was explained in light of their factor score arrays and factor structures (Focht 2002). These findings shed light on basin-wide perspectives on impacts and management preferences, which were found to be useful in conflict assessment and in informing policy deliberation. Mental modeling data were analyzed by coding responses as accurate, wrong, peripheral (off-point), particularistic (too narrow to make connections to adjacent nodes in the expert model), indeterminate (skipped nodes in the expert model), or missing (ignorance). These data were analyzed using descriptive statistics.

Finally, the ranking of concerns conducted in round three were analyzed again with descriptive statistics and compared to the concern statistics obtained in round one (content analysis of transcripts of discussions and cognitive mapping concerns). Again, good agreement among the three assessments of the identification, relative frequency, and relative importance of concerns was found. For additional information, see Focht et al. (2001), Whitaker and Focht (2001), Focht (2002). The major sociopolitical baseline findings were:

1. Disagreements existed over the magnitudes and causes of problems in the Illinois River as well as over who was to blame and what should be done.

2. Stakeholders trusted government - particularly the federal government - much less than they trusted independent experts and each other. As a result, stakeholders expressed an overwhelming preference to participate directly in the policy making process as opposed to deferring to government discretion.

3. The conflicts that existed were not bipolar. This means that although stakeholders believed that controversy and factual uncertainty were high and continuing, most were not so ideologically committed to a position that consensus was impossible.

4. Stakeholders were most concerned and knowledgeable about problems and associated impacts that were visible, local, and had received media attention - including problems with alcohol consumption, inappropriate behavior by tourists, and littering. However, policy makers were most concerned about less visible, widespread, water quality-based problems and impacts such as nutrient loading (phosphorus) from fertilizer over-application and municipal effluents and riparian area protection.

5. Stakeholders preferred coercive policies regarding enforcement of existing laws and regulations, such as applied to point source discharges and violations of civil law regarding 
behavior, drinking, and drugs. However, they preferred educational and voluntary strategies, with compensation for economic losses, for impact management from less regulated activities such as farming and other rural and suburban land uses.

\section{Watershed Management Decision Support System}

Initial efforts to visualize the simulation of water quality and other issues in the Illinois River basin involved the transformation of the digital elevation model into a data structure required by Macromedia Director software. The work on this program was designed to include four layers of information that were subsequently integrated: A flight layer, a photo layer, a water quality layer, and an aquatic life layer. The flight layer enabled a user to experience a virtual flight over the watershed with a three-dimensional effect. The photo layer enabled the user to select a certain region and have a close look at different environmental concerns. About 72 photographs with the description of each concern were taken by helicopter with the OSRC administrator. The water quality layer depicted the map of the gauging stations with historical data displayed for each station on water quality parameters, including phosphorus, nitrates, turbidity, etc. The graphs were supplemented by a description of the critical issues concerning the status of each water quality parameter. The aquatic life layer showed the number and diversity of selected species within the river system and Lake Tenkiller.

To facilitate understanding by stakeholders of the complex biological, physical, and sociopolitical relationships in the watershed, interactive influence diagrams of these systems used by the socio-political assessment team were developed along with the other visual elements (see Fig. 1). The resulting visualization vehicle was known as the Watershed Management Decision Support System (WMDSS) and was subsequently used in the policymaker and stakeholder negotiation workshops (see Fig. 2). For reference, the WMDSS also contained the most recent Illinois River Management Plan, a Riparian Area Protection Handbook, and a 20 minute video of the Illinois River Watershed and its history. For additional information, see Meo and Pettus (2001).

\section{Negotiation Workshops}

The asynchronous policy dialogue was conducted in three rounds, beginning with a 2day policymakers' workshop in October, 2000. Two professional negotiation facilitators assisted with the workshops. The policymakers initially focused on phosphorus management and riparian area protection. After an educational session, stakeholders agreed to these two priorities, but also wanted to include alcohol and behavior control. Subsequent sessions produced a consensus on these three policies by both groups. Participant judgments of policy legitimacy demonstrated the three legitimacy criteria: technical and economic effectiveness, administrative and legal implementability, and sociopolitical acceptability. Policymaker participants judged the policy dialogue to be worthwhile, trust building, and valuable to them in other policy contexts.

To test the protocol's efficacy, a random sample telephone survey of stakeholders in the basin was conducted in April, 2002. It confirmed that all provisions of the three policy proposals generated high levels of satisfaction with the phosphorus management policy achieving 95 percent satisfaction, the riparian area protection policy 91 percent, and the alcohol and behavior control policy 85 percent. The exception was two tourist fee increases that were added at the last workshop as revenue generating mechanisms despite opposition by stakeholder participants. This robust test supports the claim that the policy legitimation protocol was successful. With these results, the research team concluded that asynchronous policy dialogues that are informed by intensive assessments of stakeholders' concerns, preferences, and knowledge, can be successful in contexts dominated by distrust, controversy, and factual uncertainty.

\section{Educational, Research, and Policy Applications}

Undertaken by nine faculty members from Oklahoma's two leading research universities, the Illinois River project employed a sizeable number of undergraduate and graduate students, four of whom completed their thesis or dissertation requirements while employed by the project. While each team member contributed to the overall progress and ultimate success of the project, many elected to 


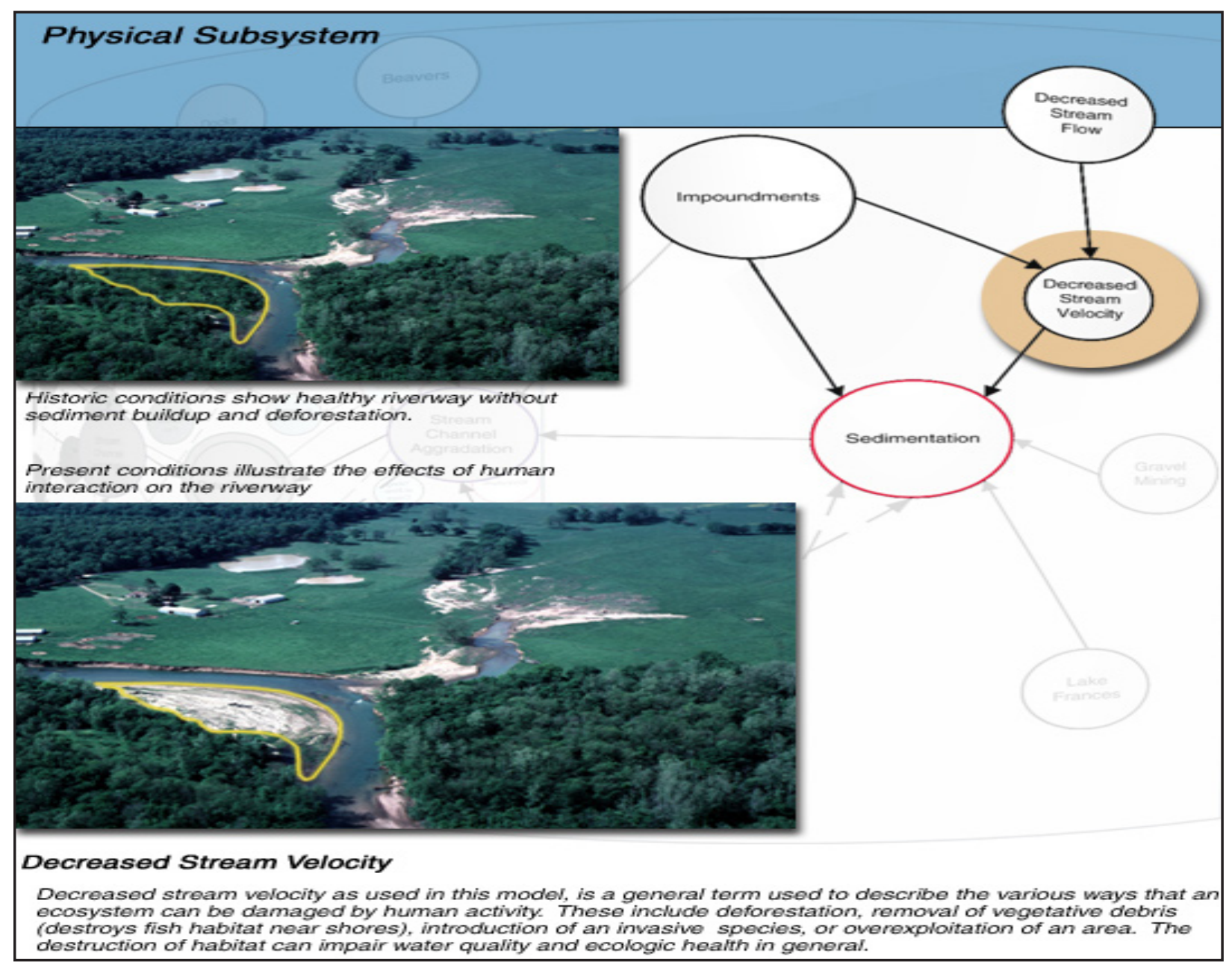

Figure 1. Site photos with influence diagram.

publish their individual research findings in the academic literature. The team leader encouraged this publication outlet since the team had earlier considered an option to combine the entire project into a book that would provide state-of-the-art information on stakeholder-based decision making in watershed planning and management. This plan, however, was shelved after technical problems prevented the final completion of the WMDSS.

Despite this setback, the EPA agreed to fund a small group of Water and Watershed project researchers, including several from the Illinois River project to assemble an academic book on stakeholder-based decision making. The book, Swimming Upstream: Collaborative Approaches to Watershed Management, was published by MIT Press in 2005, and has received a number of glowing reviews. Of the nine chapters in the book, four were coauthored by members of the Illinois
River research team (see Focht and Trachtenberg 2005, Lubell et al. 2005, Sabatier et al. 2005, Trachtenberg and Focht 2005). In addition, a special 2001 issue of Oklahoma Politics, edited by W. Focht, was dedicated to the Illinois River project in which several of the research team members published papers (see Focht et al. 2001a, Sankowski 2001, Trachtenberg 2001).

In the research area, team members from Oklahoma State University have continued to follow a vigorous program of watershed-oriented studies that combine stakeholder views and values with management options. Despite the setbacks that befell the WMDSS, W. Focht and his OSU colleagues have applied the concept of a decisionsupport system to other watersheds in Oklahoma and Arkansas. Other team faculty members, particularly B. Vieux of OU and K. Willett of OSU, have continued to develop their hydrological and 


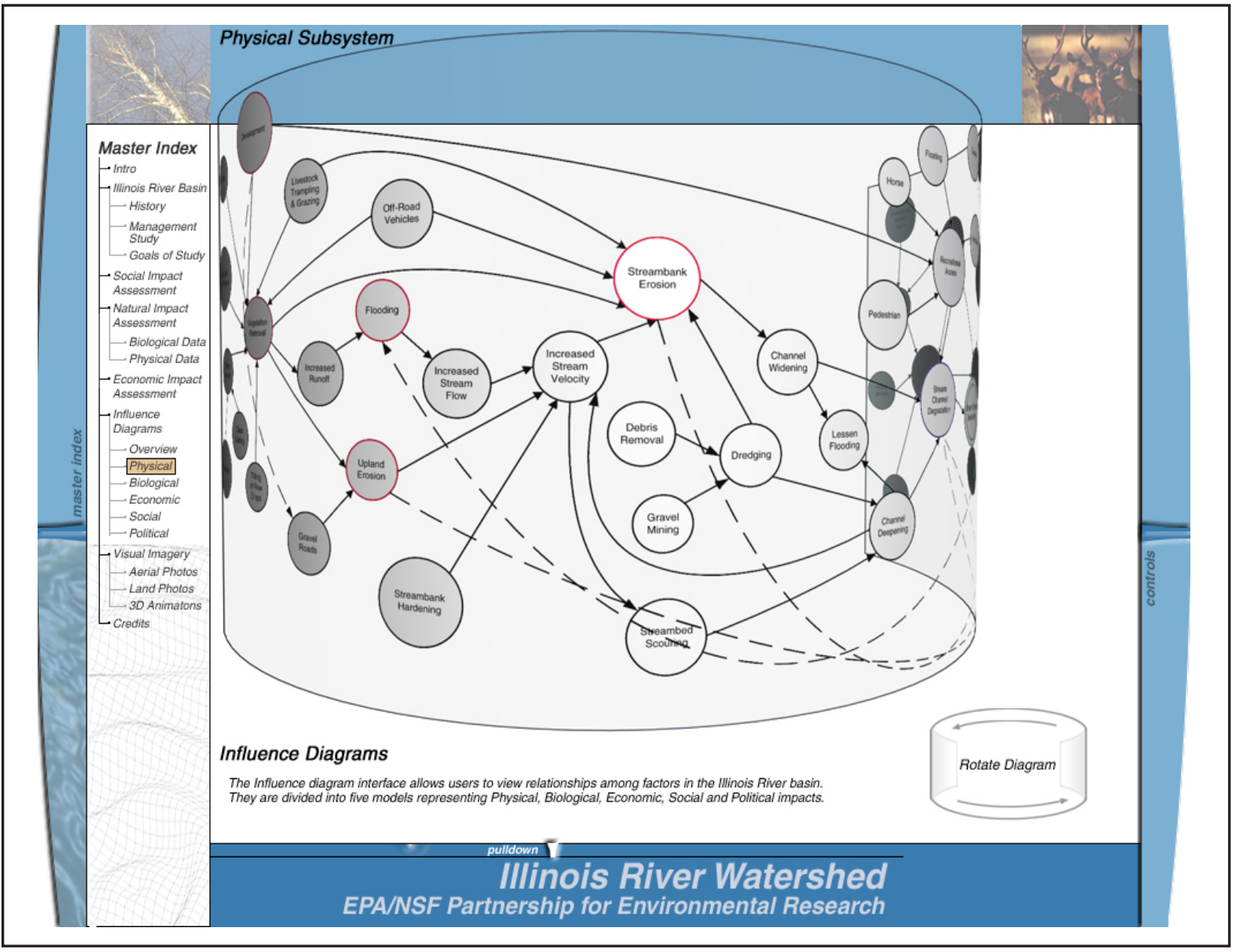

Figure 2. WMDSS interface.

economic modeling techniques with applications to environmental issues of concern.

In the realm of public policy, the stakeholderbased approach to water resources planning has had a significant impact. Based upon an earlier meeting with the research team, the Oklahoma Water Resources Board received funding from the state legislature in 2006 to undertake a five-year update of the Oklahoma Comprehensive Water Plan that will be based on stakeholder views and values. In addition, the Scenic Rivers Commission received copies of all of the project's work products and several seminars by team members on what meaning the research held for watershed management (e.g. Focht et al. 2001b). The OSRC administrator has found the information and analyses in these reports to be very useful to his management activities. Beyond the watershed, however, disagreements that had been smoldering earlier between Arkansas and Oklahoma over declining water quality in the Illinois River basin became even more contentious. During the period that the research was being completed, Oklahoma became more adamant about protecting its water quality in the transboundary river region and admonished Arkansas to reduce the amount of phosphorus that was entering receiving waters from chicken-growing operations in the basin. Against the threat of litigation, poultry producers in Arkansas agreed to participate in several mediation sessions with Oklahoma's attorney general to try to negotiate an acceptable solution. After several years, this strategy fell short of expectations, and in June, 2005 Oklahoma filed a formal lawsuit against the area's leading poultry producers. The filing cited the fact (first estimated by R. Lynch in the Illinois River project) that the amount of phosphorus from poultry waste is equivalent to the waste that would be generated by 10.7 million people, a population greater that the states of 
Arkansas, Kansas, and Oklahoma combined.

\section{Conclusions}

As a research undertaking, the Illinois River project sought to design and test the adequacy of a management protocol that was fully legitimated. In this respect, the project was a clear success. Publication of the edited book, Swimming Upstream, has drawn national attention to the quality of the research undertaken in the basin. In addition, the assemblage of multiple-disciplined researchers worked quite well as a team and in their analyses of the issues defined by the stakeholders. The inclusion of two philosophers had the benefit of providing the traditional technical experts on the team with different perspectives and insights into stake-holder based decision making. Yet the project also fell short in a few ways. First, the computer-based visualization tool (WMDSS), while extremely useful for integrating team research results, failed to live up to expectations. What was envisioned as an innovative tool for integrating scientific and technical information and making complex systems more intelligible to the lay public was not brought to fruition. Second, the limitations set on the project constrained its wider applicability to the accommodation of larger political disputes. For example, membership in the policy maker stakeholder group did not include representatives of the poultry industry in the strict sense. While it did contain members of poultry and agricultural interests, the project's funding constraints prevented the team from recruiting stakeholders from the Arkansas side of the watershed. In the future, more ambitious projects might overcome these deficiencies.

In hindsight, the Illinois River project may be reflective of the kinds of activities that the states of Arkansas and Oklahoma needed to be engaged in, only much earlier, and on a much larger scale. While it may be overly optimistic to expect an academic research project to play a significant role in the successful negotiations between two adversarial states, it is instructive that the project did address the key elements that are central to the current impasse, and may yet provide a sound basis for finding a viable path toward their resolution.

\section{Acknowledgements}

This paper is based on research supported by the EPA/NSF Water and Watersheds program through award R825791. The author thanks Ed Fite, Oklahoma Scenic Rivers Commission, and his academic colleagues: Lowell Caneday, Will Focht, Robert Lynch, Blake Pettus, Ed Sankowski, Zev Trachtenberg, Baxter Vieux, and Keith Willett. The research team expresses its thanks to Barb Levinson of the EPA and Penny Firth of the NSF for their assistance and editorial improvements.

\section{Author Bio and Contact Information}

Mark Meo is Professor of Civil Engineering and Environmental Science and a Research Fellow in Science and Public Policy at the University of Oklahoma. His research interests include environmental policy innovation, technological innovation of energy systems, watershed management and decision making, and sustainable development. Prof. Meo joined the OU faculty in 1985 after completing a 2-year postdoctoral fellowship in marine policy at the Woods Hole Oceanographic Institution. He holds a B.A. degree in biology from Northeastern University; an M.S. in marine sciences from Louisiana State University, and a Ph.D. in ecology and policy analysis from the University of California at Davis. Address: 100 East Boyd St., Room S-208, University of Oklahoma, Norman, OK 73019, Phone: 405-325-2272, FAX: 405-325-3180, Email: mmeo@ou.edu.

\section{References}

Bality, A., L. Caneday, E. Fite III, T. Wikle, and M. Yuan, 1998. Illinois River Basin Management Plan. Oklahoma Scenic Rivers Commission, Tahlequah, OK.

Focht, W., M. Langston and T. DeShong. 2001a. Informing the policy process with cognitive mapping," Oklahoma Politics 10 157-178. (special issue entitled "Environmental Policy in Oklahoma: Issues, Innovations, Insights," edited by Will Focht).

Focht, W., T. DeShong, K. Whitaker, J. Wood, and M. Langston. 2001b. Illinois River Basin Sociopolitical Impact Assessment: Stakeholder impact concerns and impact management preferences. Report to the Oklahoma Scenic Rivers Commission. 
Focht, W. 2002. Assessment and management of policy conflict in the Illinois River watershed in Oklahoma: An application of $\mathrm{Q}$ methodology. International Journal of Public Administration, 25 (11): 13111349.

Focht, W. and Z. Trachtenberg. 2005. A Trust-Based Guide to Stakeholder Participation. In Swimming Upstream: Collaborative Approaches to Watershed Management, P. Sabatier, W. Focht, Z. Trachtenberg, A. Vedlitz, and M. Matlock (eds). MIT Press: Cambridge, MA.

Lubell, M., P. Sabatier, A. Vedlitz, W. Focht, Z. Trachtenberg, and M. Matlock. 2005. Collaborative Approaches to Watershed Management. In Swimming Upstream: Collaborative Approaches to Watershed Management, P. Sabatier, W. Focht, Z. Trachtenberg, A. Vedlitz, and M. Matlock (eds). MIT Press: Cambridge, MA.

Meo, M. and B. Pettus. 2001. Computer Visualization for Integrated Watershed Management: The Illinois River Basin. In Decision Support Systems for Water Resources Management, AWRA/UCOWR Summer Specialty Conference, June 7-30.

Meo, M., W. Focht, L. Caneday, R. Lynch, F. Moreda, B. Pettus, E. Sankowski, Z. Trachtenberg, B. Vieux, and K. Willett. 2002. Negotiating science and values with stakeholders in the Illinois river basin. Journal of the American Water Resources Association 38 (2): 541-554.

Sabatier, P., W. Focht, M. Lubell, Z. Trachtenberg, A. Vedlitz, and M. Matlock. 2005. Collaborative Approaches to Watershed Management. In Swimming Upstream: Collaborative Approaches to Watershed Management, P. Sabatier, W. Focht, Z. Trachtenberg, A. Vedlitz, and M. Matlock (eds.) MIT Press: Cambridge, MA.

Sankowski, E. 2001. Environmental management and democratic legitimacy. Oklahoma Politics 10 (special issue entitled "Environmental Policy in Oklahoma: Issues, Innovations, Insights," edited by Will Focht) Oklahoma Politics 10: 21-32.

Trachtenberg, Z. 2001. Scientists and stakeholders: Evaluating the legitimacy of the Illinois river basin management protocol, Oklahoma Politics 10 (special issue entitled "Environmental Policy in Oklahoma: Issues, Innovations, Insights," edited by Will Focht) Oklahoma Politics 10: 33-44
Trachtenberg, Z. and W. Focht. 2005. Legitimacy and watershed collaborations. In Swimming Upstream: Collaborative Approaches to Watershed Management, P. Sabatier, W. Focht, Z. Trachtenberg, A. Vedlitz, and M. Matlock (eds). MIT Press: Cambridge, MA.

Vieux, B. E. and F. G. Moreda, 2002a. Ordered PhysicsBased Parameter Adjustment of a Distributed Model. Chapter 20 in Advances in Calibration of Watershed Models, Q. Duan, S. Sorooshian, H.V. Gupta, A.N. Rousseau, R. Turcotte, (eds). Monograph Series on Water Resources, American Geophysical Union, ISBN 0-87590-355-X, pp. 267-281.

Vieux, B. E. and F. G. Moreda, 2002b. Densification of nutrient concentration and loading for simulation of management alternatives in the Illinois River Basin. Eos: Transaction of the American Geophysical Union 83(19), Spring Meeting. Suppl., Abstract H42D-22.

Vieux, B. E. and F. G. Moreda, 2002c. Assessing the hydrologic worth of spatial variability using a distributed hydrologic model of the Illinois River basin. Eos: Transaction of the American Geophysical Union 83(19), Spring Meet. Suppl., Abstract H51B07.

Vieux, B. E. and F. G. Moreda. 2003. Nutrient loading assessment in the Illinois River using a synthetic spproach. Journal of the American Water Resources Association 39 (4): 757-769.

Whitaker, K. and W. Focht. 2001. Expert modeling of environmental impacts. Oklahoma Politics 10: PAGES (special issue entitled "Environmental Policy in Oklahoma: Issues, Innovations, Insights," edited by Will Focht) Oklahoma Politics 10: 179-186.

Willett, K., D. M. Mitchell, H. L. Goodwin, B. Vieux, and J. S. Popp,. 2006. The Opportunity Cost of Regulating Phosphorus from Broiler Production in the Illinois River Basin, Journal of Environmental Planning and Management 49 (2): 181-20. 